\title{
Insights into the Transport Properties of
}

\section{Electrolyte Solutions in a Hierarchical Carbon}

\section{Electrode by Molecular Dynamics Simulations}

Mostafa Elabyouki ${ }^{1}$, Daniel Bahamon ${ }^{1,2}$, Maryam Khaleel ${ }^{1,2}$ and Lourdes F. Vega ${ }^{1,2, *}$

${ }^{1}$ Chemical Engineering Department, Khalifa University, P.O. Box 127788, Abu Dhabi, UAE

${ }^{2}$ Research and Innovation Center on $\mathrm{CO}_{2}$ and $\mathrm{H}_{2}(\mathrm{RICH})$ and Center for Catalysis and Separation (CeCaS), Khalifa University, P.O. Box 127788, Abu Dhabi, UAE

\section{Supporting Information}

Table S1. Non-bonded force field parameters employed in this work.

\begin{tabular}{|c|c|c|c|c|c|}
\hline Molecule & Atom & $\sigma(\dot{\mathbf{A}})$ & $\varepsilon$ (kcal/mol) & $q(e)$ & Ref \\
\hline \multirow{5}{*}{ Graphene } & Normal C & 3.40 & 0.0556 & 0.0 & \multirow[t]{5}{*}{ (1) } \\
\hline & Carbonyl C & 3.75 & 0.105 & 0.55 & \\
\hline & Carbonyl O & 2.96 & 0.210 & -0.5 & \\
\hline & Hydroxyl O & 3.07 & 0.155 & -0.64 & \\
\hline & $\mathrm{H}$ & 0.0 & 0.0 & 0.45 & \\
\hline \multirow[t]{2}{*}{ Nitrogen } & $\mathrm{N}$ & 3.31 & 0.0715 & -0.482 & \multirow[t]{2}{*}{ (2) } \\
\hline & $\mathrm{COM}$ & - & - & 0.964 & \\
\hline \multirow[t]{3}{*}{$\mathrm{LiPF}_{6}$} & $\mathrm{Li}$ & 1.4424 & 0.10314 & 1.0 & \multirow[t]{3}{*}{ (3) } \\
\hline & $\mathrm{P}$ & 3.695 & 0.13169 & 1.07 & \\
\hline & $\mathrm{F}$ & 2.9347 & 0.028716 & -0.345 & \\
\hline \multirow[t]{4}{*}{ DMSO } & $\mathrm{C}$ & 4.08 & 0.078 & -0.148 & \multirow[t]{4}{*}{ (4) } \\
\hline & $\mathrm{H}$ & 2.68 & 0.024 & 0.090 & \\
\hline & $\mathrm{S}$ & 4.00 & 0.350 & 0.312 & \\
\hline & $\mathrm{O}$ & 3.40 & 0.120 & -0.556 & \\
\hline
\end{tabular}

Table S2: MD overall diffusivity results for $\mathrm{Li}^{+}, \mathrm{PF}_{6}-$ and $\mathrm{DMSO}$.

\begin{tabular}{|l|l|l|l|}
\hline System Charge & $\mathbf{L i}^{+}$Diffusivity & $\mathbf{P F}_{6}{ }^{-}$Diffusivity & DMSO Diffusivity \\
\hline
\end{tabular}




\begin{tabular}{|c|c|c|c|}
\hline $\mathbf{( - e )}$ & $\left.\mathbf{( 1 0}^{-\mathbf{8}} \mathbf{c m}^{2} / \mathbf{s}\right)$ & $\left.\mathbf{( 1 0}^{-8} \mathbf{c m}^{2} / \mathbf{s}\right)$ & $\left.\mathbf{( 1 0}^{-8} \mathbf{c m}^{\mathbf{2}} / \mathbf{s}\right)$ \\
\hline $\mathbf{0}$ & $2440 \pm 0.05 \%$ & $26047 \pm 0.04 \%$ & $8250 \pm 0.012 \%$ \\
\hline $\mathbf{5}$ & $103 \pm 0.14 \%$ & $139 \pm 0.15 \%$ & $34975 \pm 0.12 \%$ \\
\hline $\mathbf{1 0}$ & $25.7 \pm 0.08 \%$ & $99.6 \pm 0.05 \%$ & $17798 \pm 0.02 \%$ \\
\hline $\mathbf{2 0}$ & $2.59 \pm 0.45 \%$ & $57.6 \pm 0.7 \%$ & $3940 \pm 0.06 \%$ \\
\hline
\end{tabular}

Table S3: MD diffusivity results in the $z$-direction for $\mathrm{Li}^{+}, \mathrm{PF}_{6}{ }^{-}$and DMSO.

\begin{tabular}{|c|c|c|c|}
\hline $\begin{array}{c}\text { System Charge } \\
\text { (-e) }\end{array}$ & $\begin{array}{c}\mathrm{Li}^{+} \text {Diffusivity-z } \\
\left(10^{-9} \mathrm{~cm}^{2} / \mathrm{s}\right)\end{array}$ & $\begin{array}{c}\text { PF }_{6}-\text { Diffusivity }-z \\
\left(10^{-9} \mathrm{~cm}^{2} / \mathrm{s}\right)\end{array}$ & $\begin{array}{l}\text { DMSO Diffusivity-z } \\
\qquad\left(10^{-9} \mathrm{~cm}^{2} / \mathrm{s}\right)\end{array}$ \\
\hline $\mathbf{0}$ & $306 \pm 0.10 \%$ & $1198 \pm 0.18 \%$ & $1465 \pm 0.1 \%$ \\
\hline 5 & $17.8 \pm 0.3 \%$ & $79.3 \pm 0.6 \%$ & $4113 \pm 0.4 \%$ \\
\hline 10 & $9.1 \pm 0.4 \%$ & $178 \pm 0.07 \%$ & $1387 \pm 0.1 \%$ \\
\hline 20 & $5.5 \pm 0.3 \%$ & $418 \pm 0.17 \%$ & $1878 \pm 0.4 \%$ \\
\hline
\end{tabular}

Table S4: Comparison between $\mathrm{Li}^{+}$ion diffusivity in this work and literature.

\begin{tabular}{|c|c|c|c|c|}
\hline $\begin{array}{l}\mathrm{Li}^{+} \text {overall } \\
\text { diffusivity } \\
\left(\mathrm{cm}^{2} / \mathrm{s}\right)\end{array}$ & $\begin{array}{c}\mathbf{L i}^{+} z_{-} \\
\text {direction } \\
\text { diffusivity } \\
\left(\mathrm{cm}^{2} / \mathbf{s}\right)\end{array}$ & Morphology & Method & Reference \\
\hline $\begin{array}{l}2.6 \times 10^{-8}- \\
2.4 \times 10^{-5}\end{array}$ & $\begin{array}{l}3.1 \times 10^{-7}- \\
5.5 \times 10^{-9}\end{array}$ & $\begin{array}{l}\text { Hierarchical graphene } \\
\text { structure }\end{array}$ & MD & This work \\
\hline $4.4 \times 10^{-6}$ & $8.7 \times 10^{-12}$ & $\begin{array}{l}\text { Highly oriented pyrolytic } \\
\text { graphite (HOPG) }\end{array}$ & $\begin{array}{l}\text { Chronoampero- } \\
\text { metry and ab initio } \\
\text { kMC simulations }\end{array}$ & $\begin{array}{l}\text { Persson et } \\
\text { al. } 5\end{array}$ \\
\hline $1.35 \times 10^{-10}$ & & $\begin{array}{l}\text { Spherical graphite } \\
\text { particles }\end{array}$ & EIS & Yu et al. ${ }^{6}$ \\
\hline $10^{-7}-10^{-6}$ & & Doped graphene sheets & $\mathrm{CV}$ & $\mathrm{Wu}$ et al. ${ }^{7}$ \\
\hline $10^{-8}-10^{-6}$ & & $\mathrm{LiCoO}_{2}$ & $\begin{array}{l}\text { Atomic force } \\
\text { microscopy }\end{array}$ & Balke et al. ${ }^{8}$ \\
\hline $\begin{array}{l}5.0 \times 10^{-9}- \\
1.0 \times 10^{-8}\end{array}$ & & Graphite & MD & $\begin{array}{l}\text { Márquez et } \\
\text { al. }{ }^{9}\end{array}$ \\
\hline
\end{tabular}


Table S5: MD overall diffusivity results for the electrolyte solution at various $\mathrm{Li}^{+}$ion concentrations.

\begin{tabular}{|c|c|c|c|}
\hline $\begin{array}{l}\mathrm{Li}^{+} \text {Concentration } \\
\quad(\mathrm{mol} / \mathrm{cm} 3)\end{array}$ & $\begin{array}{c}\mathbf{L i}^{+} \text {Diffusivity } \\
\left(10^{-8} \mathrm{~cm}^{2} / \mathrm{s}\right)\end{array}$ & $\begin{array}{c}\text { PF }_{6}-\text { Diffusivity } \\
\left(10^{-8} \mathrm{~cm}^{2} / \mathrm{s}\right)\end{array}$ & $\begin{array}{l}\text { DMSO Diffusivity } \\
\qquad\left(10^{-8} \mathrm{~cm}^{2} / \mathrm{s}\right)\end{array}$ \\
\hline 0.00175 & $25.7 \pm 0.08 \%$ & $99.6 \pm 0.05 \%$ & $17798 \pm 0.02 \%$ \\
\hline 0.00211 & $339 \pm 0.03 \%$ & $761 \pm 0.04 \%$ & $17798 \pm 0.012 \%$ \\
\hline 0.00282 & $662 \pm 0.6 \%$ & $765 \pm 0.07 \%$ & $31028 \pm 0.11 \%$ \\
\hline
\end{tabular}

Table S6: MD diffusivity results in the $z$-direction for the electrolyte solution at various $\mathrm{Li}^{+}$ion concentrations.

\begin{tabular}{|c|c|c|c|}
\hline $\begin{array}{c}\mathrm{Li}^{+} \text {Concentration } \\
(\mathrm{mol} / \mathrm{cm} 3)\end{array}$ & $\begin{array}{c}\mathrm{Li}^{+} \text {Diffusivity- } z \\
\left(10^{-9} \mathrm{~cm}^{2} / \mathrm{s}\right)\end{array}$ & $\begin{array}{c}\mathrm{PF}_{6}-\text { Diffusivity- } z \\
\left(10^{-9} \mathrm{~cm}^{2} / \mathrm{s}\right)\end{array}$ & $\begin{array}{c}\text { DMSO Diffusivity- } z \\
\left(10^{-9} \mathrm{~cm}^{2} / \mathrm{s}\right)\end{array}$ \\
\hline 0.00175 & $9.14 \pm 0.4 \%$ & $178 \pm 0.07 \%$ & $1387 \pm 0.06 \%$ \\
\hline 0.00211 & $585 \pm 0.15 \%$ & $2219 \pm 0.11 \%$ & $38814 \pm 0.02 \%$ \\
\hline 0.00282 & $2132 \pm 0.6 \%$ & $2253 \pm 0.6 \%$ & $46681 \pm 0.20 \%$ \\
\hline
\end{tabular}



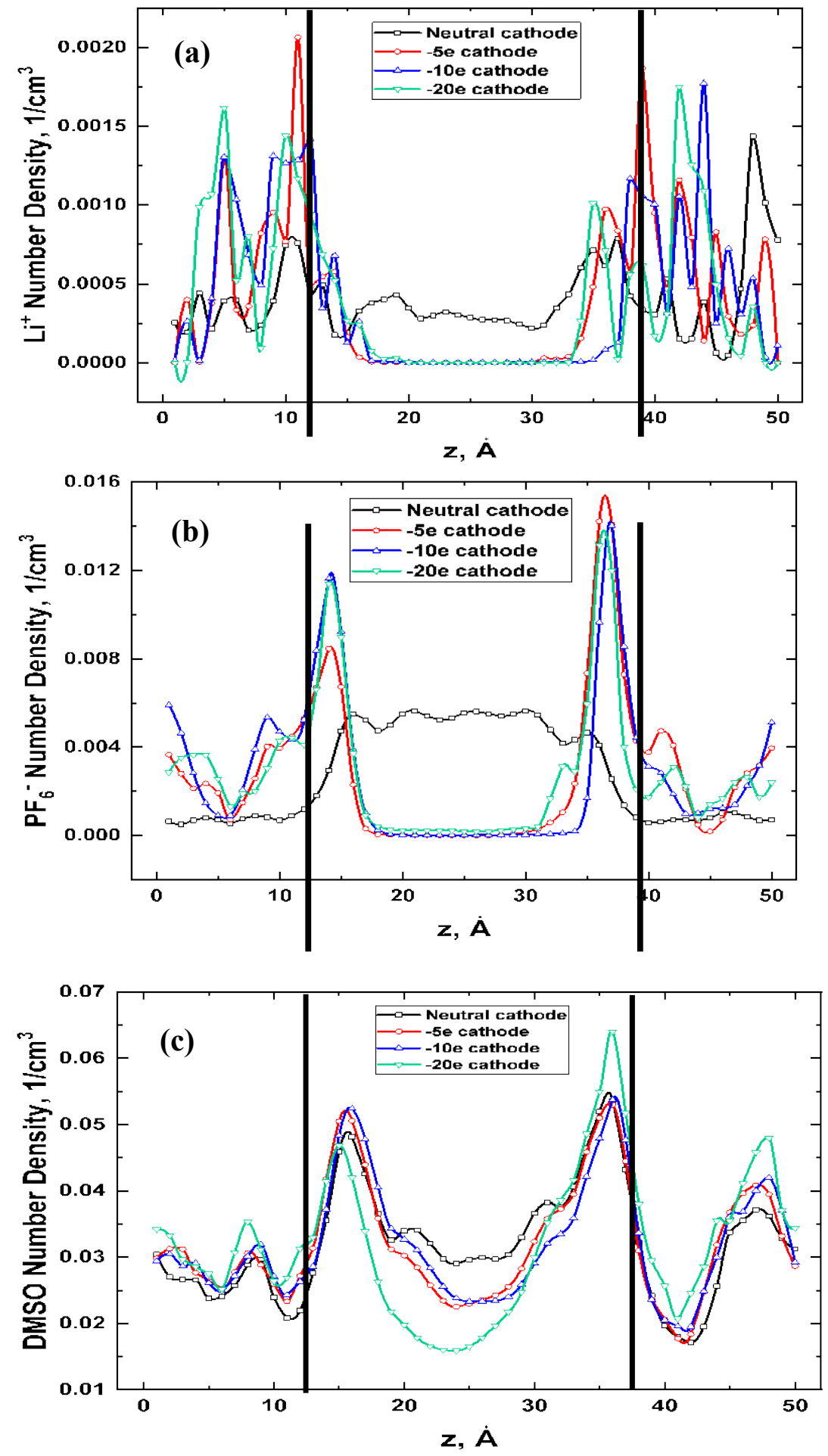

Figure S1: Combined density profiles under various cathodic charges. (a) $\mathrm{Li}^{+}$, (b) $\mathrm{PF}_{6}{ }^{-}$, (c) DMSO. 


\section{References}

1. Jorge, M.; Schumacher, C.; Seaton, N. A. Simulation Study of the Effect of the Chemical Heterogeneity of Activated Carbon on Water Adsorption. Langmuir 2002, 18 (24), 92969306.

2. Siepmann, J. I.; Frenkel, D. Configurational Bias Monte Carlo: A New Sampling Scheme for Flexible Chains, Mol. Phys. 1992, 75, 59-70.

3. Kumar, N.; Seminario, J. Lithium-Ion Model Behavior in an Ethylene Carbonate Electrolyte using Molecular Dynamics. J. Phy. Chem. C 2016, 120, 16322-16332.

4. Strader, M.; Feller, S. A Flexible All-Atom Model of Dimethyl Sulfoxide for Molecular Dynamics Simulations. J. Phy. Chem. A 2002, 106, 1074-1080.

5. Persson, K., Sethuraman, V.; Hardwick, L.; Hinuma, Y.; Meng, Y.; Van Der Ven, A.; Srinivasan, V.; Kostecki, R.; Ceder, G. Lithium Diffusion in Graphitic Carbon. J. Phys. Chem. Lett. 2010, 1, 1176-1180.

6. Yu, P.; Popov, B. N.; Ritter, J. A.; White, R. E. Determination of the Lithium Ion Diffusion Coefficient in Graphite. J. Electrochem. Soc. 1999, 146, 8-14.

7. Wu, Z.-S.; Ren, W.; Xu, L.; Li, F.; Cheng, H.-M. Doped Graphene Sheets As Anode Materials with Superhigh Rate and Large Capacity for Lithium Ion Batteries. ACS Nano 2011, 5, 54635471.

8. Balke, N.; Jesse, S.; Morozovska, A.; Eliseev, E.; Chung, D.; Kim, Y.; Adamczyk, L.; Garcia, R.; Dudney, N.; Kalinin, S. Nanoscale Mapping of Ion Diffusion in a Lithium-Ion Battery Cathode. Nat. Nanotechnol. 2010, 5, 749-754.

9. Márquez, A.; Balbuena, P. B. Molecular Dynamics Study of Graphite/Electrolyte Interfaces. J. Electrochem. Soc. 2001, 148, 624-635. 